\title{
Privacy in public and the contextual conditions of agency
}

\section{Maria Brincker ${ }^{1}$}

\begin{abstract}
:
As traditional public spaces rapidly change the question arises whether pervasive surveillance is actually compatible with the nature of individual agency. It is known that new and largely unregulated technologies often both erode old de facto limitations of access, and erect new asymmetric barriers of knowledge, but how should we understand the harms of these new contexts? Based on our psychological and biological knowledge of social perception, embodiment and action choice it seems that these conditions present a toxic mix for our ability to gauge what I call our "relational privacy", and thereby for our autonomous and purposeful action planning. If this functional analysis is right then boundless surveillance of our bodies and behaviors threatens rather than supports basic personal freedom and responsibility. However, in contrast to this analysis, the private-public dichotomy often implied in existing debates assumes that privacy concerns can be theorized without much attention to the functional and relational dynamics of our embodied agency. Building on among others Nissenbaum and Reidenberg's work, this chapter seeks to counter this tendency. Two conclusions stand out: Firstly, it seems that our action choices depend on the existence of limited contexts also in public, and thus actions in what we might term "unbounded contexts" are typically performed through some kind of denial. Secondly, abundant availability of data traceable to persons can not only undermine and coerce our choices, but even to a large extent completely bypass our current volitional action choices. Thus it seems that the contextual nature of our agency should be of utmost importance to political and legal privacy issues, and could usefully guide attempts to re-create more bounded public spaces.
\end{abstract}

\section{Introduction}

Current technology and surveillance practices make behaviors traceable to persons in unprecedented ways. This causes a loss of anonymity and of many privacy measures relied on in the past. These de facto privacy losses are by many seen as problematic for individual psychology, intimate relations and democratic practices such as free speech and free assembly. ${ }^{2}$ I share most of these concerns but propose that an even more fundamental problem might be that our very ability to act as autonomous and purposive agents relies on some degree of privacy, perhaps particularly as we act in public and semi-public spaces.

I suggest that basic issues concerning action choices have been left largely unexplored, due to a series of problematic theoretical assumptions at the heart of privacy debates. One such assumption has to do with the influential conceptualization of privacy as pertaining to personal intimate facts belonging to a private sphere as opposed to a public sphere of public facts. As Helen Nissenbaum has pointed out, the notion of privacy in public sounds almost like an oxymoron given this traditional private-public dichotomy. ${ }^{3}$ I discuss her important attempt to defend privacy in public through her concept of 'contextual integrity.' Context is crucial, but Nissenbaum's descriptive notion of existing norms seems to fall short of a

\footnotetext{
${ }^{1}$ I thank Mickaella Perina and David Flesche for discussions helping me write this chapter. Thanks also to the students in my 2014-16 classes at UMass Boston.

${ }^{2}$ See e.g. Titus Stahl, 'Indiscriminate mass surveillance and the public sphere' (2016) 18 Ethics and Information Technology 33, and Joel Reidenberg, 'Privacy in Public' (2014) 69 U Miami L Rev 141, discussed later.

${ }^{3}$ Helen Nissenbaum 'Toward an approach to privacy in public: Challenges of information technology' (1997) 7 Ethics \& Behavior 207.
} 
solution. I here agree with Joel Reidenberg's recent worries regarding any approach that relies on 'reasonable expectations" ${ }^{4}$. The problem is that in many current contexts we have no such expectations. Our contexts have already lost their integrity, so to speak.

By way of a functional and more biologically inspired account, ${ }^{5} \mathrm{I}$ analyze the relational and contextual dynamics of both privacy needs and harms. Through an understanding of action choice as situated and options and capabilities as relational, a more consequence-oriented notion of privacy begins to appear. I suggest that privacy needs, harms and protections are relational. Privacy might have less to do with seclusion and absolute transactional control than hitherto thought. It might instead hinge on capacities to limit the social consequences of our actions through knowing and shaping our perceptible agency and social contexts of action. To act with intent we generally need the ability to conceal during exposure. If this analysis is correct then relational privacy is an important condition for autonomic purposive and responsible ${ }^{6}$ agency_-particularly in public space.

Overall, this chapter offers a first stab at a reconceptualization of our privacy needs as relational to contexts of action. In terms of 'rights to privacy' this means that we should expand our view from the regulation and protection of the information of individuals to questions of the kind of contexts we are creating. I am here particularly interested in what I call 'unbounded contexts', i.e. cases of context collapses, hidden audiences and even unknowable future agents. ${ }^{7}$

\section{Privacy in public - the private-public dichotomy reconsidered}

The nature of privacy is notoriously contested, as are the according needs, rights and harms. Many have, from the early work of Warren and Brandeis ${ }^{8}$ and onwards, attempted to define privacy as pertaining to something like a 'private sphere' as opposed to a 'public sphere'. Privacy breaches, according to such a dichotomy, have to do with personal facts belonging to a private sphere being inappropriately publicized, i.e. made public. This conceptualization gives rise to questions about the demarcation of such spheres and facts and about proper/improper publications. The latter is often described in terms of consent and contracts, and rights somewhat analogously to ownership and control. ${ }^{9}$ However a further question is whether any private-public dichotomy can account for our actual privacy needs, harms and concerns - including how we might conceive of privacy in public. Some theorists, such as Solove, suggest giving up on a unified conceptualization and rather treat privacy as a Wittgensteinian 'family resemblance' of loosely related issues. ${ }^{10}$ Others propose rethinking privacy, such that privacy practices in public can be understood. I follow this latter path and discuss respectively the proposals of Nissenbaum and Reidenberg as each in my view move this project forward and yet each retain problematic assumptions.

\subsection{Context integrity, norms and expectations}

Nissenbaum has repeatedly pointed out that, given the classic private-public dichotomy, it seems we can expect no privacy having entered ourselves or our data into public space. However, she argues, that having our behavior in public be completely 'up for grabs' seem

\footnotetext{
${ }^{4}$ Reidenberg (n 2).

${ }^{5}$ Peter H Klopfer and Daniel I Rubenstein, 'The concept privacy and its biological basis' (1977) 33 Journal of Social Issues 52.

${ }^{6}$ Note that the focus is not legal liability, but rather the conditions of moral responsibility. The goal is to discuss privacy as it relates to our social and embodied agency, and I shall largely leave questions about existing legal frameworks out and rather focus on the normative question of what kind of protections we might want or need.

${ }^{7}$ danah boyd, Taken out of context: American teen sociality in networked publics (ProQuest 2008).

${ }^{8}$ Samuel D Warren and Louis D Brandeis, 'The right to privacy' [1890] Harv L Rev 193.

${ }^{9}$ See e.g. ibid

${ }^{10}$ Daniel L Solove, 'Conceptualizing Privacy’ [2002] Cal L Rev 1087.
} 
inconsistent with our actual actions and expectations in public spaces. She writes that this would go against our intuitive "norms of privacy'" and would leave us incapable of dealing with today's most urgent privacy concerns, having to do with how our publically shared information is stored, analyzed and used for secondary purposes.

"Our revolution will not be in gathering data-don't look for TV cameras in your bedroom - but in analyzing the information that is already willingly shared." Hunter's comment makes an almost paradoxical point: We are complicit in an invasion of our own privacy that ultimately we find objectionable. The invasion is not from the realm of the intimate but from the realm that is generally not given serious consideration by many noted theorists of privacy. ${ }^{12}$

Nissenbaum proceeds to reject the traditional 'private-public dichotomy' and proposes instead an alternative account of privacy as pertaining to our norms and expectations of privacy in various spatial and functional contexts. In her own words:

At root, I believe, is a mismatch between intuitively held privacy norms as applied to information and the much touted private public dichotomy. A promising alternative rejects the relevance of the dichotomy to information about persons in favor of the idea of a multiplicity of contexts. Information learned in one context belongs in that context and is public vis-à-vis that context. We do not have a dichotomy of two realms but a panoply of realms; something considered public in relation to one realm may be private in relation to another, "disclosure of information to groups, even potentially large groups, might still be considered private provided still larger groups were excluded" (Schoeman, 1984). People count on this contextual integrity as an effective protection of privacy. Nightclub patrons may not mind being seen by other patrons but may reasonably object to having their actions reported outside of that context. ${ }^{13}$

Thus privacy according to Nissenbaum has to do with how we are to treat information related to persons given the kind of context this information is produced in and pertains to. I agree with Nissenbaum's critique of the private-public dichotomy and in terms of her positive alternative I am enthusiastic about her differentiation of multiplicities of social contexts and her introduction of the key concept of 'contextual integrity'. ${ }^{14}$ It cannot be stressed enough how most theorists in one way or another end up ignoring the contextual relationality of privacy issues. This failure has to do with the broader tendency particularly pervasive in privacy and copyright law to take subjectivity as a stable given, and thus exclude any analysis of the role of context for our lives, minds and ability to act. ${ }^{15}$ By contrast Nissenbaum highlights how we 'count on this contextual integrity as an effective protection of

\footnotetext{
11 'In general, even if we agree that a number of familiar places are not part of the "intimate" and private realms, we would not therefore agree that any information harvested from them is completely public. This would mean that facts gleaned from arenas such as public schools, supermarkets, parks, and libraries belong in a category of public information in the strongest sense. By contrast, even quintessential public places - a public square or sidewalk - are governed by some norms of privacy.' Nissenbaum (n 3) 214.

12 ibid 210.

13 ibid 215.

${ }^{14}$ Note also her important work on developing these ideas in regard to current policy issues. See e.g. Helen Nissenbaum, 'Respecting context to protect privacy: Why meaning matters' [2015] Science and engineering ethics 1 .

${ }^{15}$ For an interesting feminist critique of the mind-body dualism in copyright law see e.g. Dan L Burk, 'Feminism and dualism in intellectual property' (2007) 15 American University Journal of Gender, Social Policy \& the Law 6.
} 
privacy. ${ }^{16}$ However, when looking at the details of what she takes contextual integrity to be, I wonder if her account needs some tweaking. In the quote above Nissenbaum says that information learned in a given context 'belongs in that context'. This sounds like a general critique of aggregation, big data analytics and other practices of data harvesting for secondary use. One might take the implication that some current digital contexts - based on such secondary use - do not have appropriate 'integrity.' However Nissenbaum actually seems to shy away from this sort of normative critique of particular contexts, and instead defines 'contextual integrity' as an adherence to norms regarding that context. Thus she ends up judging integrity not by the functional value of a given context but rather through a descriptive notion of our existing 'norms' and 'expectations'.

The question is whether actual expectations can serve as the foundation for evaluations of privacy breaches. What if what is taken to be normal is not good or functional in important respects? What if it is expected that NSA uses warrantless wiretapping, or that Facebook, Google or E-ZPass will share our information and continuously change their small font policies in ways practically unknowable? What if the current norms of information sharing - as I suggest - undermine our conditions of agency, freedom, morality and democracy? In other words what if our current public contexts and current norms are dysfunctional? It seems that Nissenbaum with the descriptive notion precludes a normative critique of what has become the new normal.

\subsection{Beyond 'reasonable expectation'}

Reidenberg has recently articulated some concerns about relying on norms and aims his criticism straight at the core legal notion of 'reasonable expectation of privacy:'

In the face of "ambient surveillance," how can any notion of a reasonable expectation of privacy survive? Even the notion that a boundary can be drawn around whether technology to assist discovering information is in general use or not in general use becomes irrelevant. Alternate data sources abound. ${ }^{17}$

In the information society, the question thus becomes if it is reasonable to have an expectation of privacy at all. And hence how we can talk about a privacy problem when no 'breach' can be pointed to, because our information is gleaned from 'plain view', third parties and from multiple sources? Reidenberg highlights how the sheer abundance of data makes regulation based on tracing sources to contexts of sharing impotent and irrelevant. The issue is that these do not protect us against what he takes to be the harm of 'non-breach breaches' of privacy when information is already in some respect public. He talks about the problem of having lost 'practical' privacy protections:

...before the ubiquitous deployment of information technologies, obscurity of information provided an important degree of privacy protection. As a practical matter, data that was inaccessible was private, and the public could have expectations of actual privacy, even if theoretically the information was available for scrutiny. ${ }^{18}$

For our purposes of understanding the relation between agency and privacy, Reidenberg's practical focus is intriguing. He continues:

Before digital cameras could capture high-resolution images at great distances and

\footnotetext{
${ }^{16}$ Nissenbaum (n 3) 215.

${ }^{17}$ Reidenberg (n 4) 146-7.

18 ibid 148 .
} 
computer algorithms could match photos with identities, individuals walking through public places, like Grand Central Terminal in New York City, at rush hour, would be anonymous in a crowd. This anonymity existed even though the individuals were in plain view. Now, with surveillance cameras on every building and street corner, and with digital facial recognition capabilities in wide use on laptops and on social networks like Facebook, society can no longer claim any expectation of anonymity in crowds. ${ }^{19}$

He thus recounts how anonymity in public has been replaced with widespread accessibility. The question that needs answering however is why we need some level of 'practical privacy', what is the functional role of such privacy measures of inaccessibility etc. that he tacitly relies on? We shall return to the functionality of 'expectations of anonymity', i.e. the advantage of being faceless in a crowd, and how this is lost for instance when analyzed by facial recognition software. But what, in terms of Reidenberg's own theory, are the harms of access? Disappointingly, it seems that Reidenberg ends up taking us back to a refurbished version of the dichotomy of the private and public. He writes:

The recreation of privacy in public suggests that the "reasonable expectation of privacy" standard needs to give way to a standard that takes into consideration a variant of what Helen Nissenbaum coins as "contextual integrity." The variant is to examine the generation of personal information in order to distinguish between observable acts that are "non-public," or private-regarding, and those that are of public significance, or "governance-related." The distinction means that the nature of the act places information into the true public sphere rather than the observability of the act. ${ }^{20}$

Reidenberg's proposal thus is to distinguish a 'true public sphere' of 'governance-related' from 'private-regarding' acts and facts, and seek some kind of protection for the latter. But how does this distinction work? It seems that we need not just Nissenbaum's descriptive norms but also rules for what counts as private-regarding and public-regarding behavior and facts - as well as about what we can do with which kinds of information. He thereby avoids having privacy depend on how and where the information is obtained - whether in classically private or public space. However, I do not see how his fact-based distinction is a variant of 'contextual integrity' or how it solves our practical or theoretical obstacles.

First there is the question of how the distinction would be applied in concrete cases of surveillance, analytics and accessibility. It seems that part of the problem precisely is that these technologies make the private- and public-regarding, the intimate and the professional/political slippery and contested. ${ }^{21}$ Reidenberg talks about how contexts without anonymity are practically unmanageable for individuals. I emphatically agree, but where did the actual individuals go in his theory of the truly private and truly public? From what perspective are we to distinguish and judge these realms? It seems that he assumes some sort of consensus, or rules without rule-makers, which given our history I find hard to believe. ${ }^{22}$ The question of what we should hide and what we should reveal is both political and personal - and this fact is of course ironically intimately related to our very need for privacy.

To formulate my criticism and alternative proposal we need to take a detour around

\footnotetext{
19 ibid 149.

20 ibid 155 .

${ }^{21}$ We see this problem of the now massive commodification of the personal already in Warren and Brandeis (n 8) as they complain that personal data/gossip has become a trade with an eager market.

${ }^{22}$ The examples in Thomas Nagel's 'Exposure \& Concealment' (1998) 27 Philosophy \& Public Affairs 3, illustratively shows how what he takes to be 'neutral' norms are maintaining the affordance structure neatly fitting white-cis-male conveniences.
} 
first the nature of situated agency in general and the function of privacy in animals. Through these detours I hope to set aside the private-public dichotomy and allow us to better understand our privacy-seeking behaviors and their embodied and embedded contextual dynamics.

\section{Dynamic affordance spaces and situated action}

Life might be a string of choices - but how do we actually decide what to do at every moment? To choose any concrete action in any particular situation, we need to judge some possibilities of this situation for a person with our specific abilities, needs, values and goals. The term 'affordance' was introduced by James J. Gibson to capture the functionality and opportunities of environments in relation to agents of certain needs and capacities. 'The affordances of the environment are what it offers the animal, what it provides or furnishes, either for good or ill., ${ }^{23}$ What we consider to be our situated aspirations, options, needs and fears are crucial to our actions, and we never act from nowhere or towards nowhere. Further, our choices take place in relation not just to individual affordances but to the overall perceived and interrelated possibilities, what I call the affordance space. ${ }^{24}$

If we think of each context as an affordance space, then it becomes clear how we via active measures - policy-based or not - can change the overall affordance relations and free ourselves from certain needs, fears and conflicts of interest. Yet, when conceptualizing these as affordance changes, we suffer no illusion that we have become disembodied and nonperspectival in our outlook. Thus if I am relatively financially safe, if I live in a community with social services and infrastructures, with civil rights, with relative equality and peace, then that allows me to disregard a whole host of 'could-be-dangers' and engage in a certain set of domestic, social and political actions that would leave one extremely vulnerable under different conditions.

I suggest that our need for privacy is deeply connected to our attempt to control the social consequences of our situated embodied and embedded actions. Rational choice theorists say we 'optimize' our choices given 'utility expectations. ${ }^{\text {,25 }}$ Though many assumptions of such 'homo economicus' frameworks are questionable, the point is well taken that to make informed decisions we need some habitual expectations about the likely consequences of our actions. This is particularly important for social consequences directly traceable to one's person. However, our current largely unregulated digitized worlds of surveillance, collapsed contexts, hidden audiences and secondary re-purposing of data, ${ }^{26}$ in many ways represent what I call 'unbounded contexts'. Such contexts precisely do not generally allow us to make reasonable judgments about the actual traceable outcomes of our behavior. These technology-enhanced contexts force us instead to trust unknown agents and algorithms, which are uncertain and often precisely untrustworthy.

With the notion of an affordance space we can now think about our privacy needs and the notion of 'contextual integrity' in more value-laden and purposive terms. Nissenbaum describes contextual integrity as respect for existing local norms pertaining to specific contexts, and which therefore allows for reasonable expectations that they will be abided by. But what if we instead analyze a context in terms of its affordances and what it makes feasible in terms of actions. I suggest we then open up for different kinds of political and regulatory discussions, that can include a view to broader human conditions and needs issues. It might

\footnotetext{
${ }^{23}$ James J Gibson, 'The theory of affordances' in Robert Shaw and John Bransford (eds), Perceiving, acting, and knowing: Toward an ecological psychology (Erlbaum 1977) 67.

${ }^{24}$ Erik Rietveld and Julian Kiverstein 'A rich landscape of affordances' (2014) 26 Ecological Psychology 325.

${ }^{25}$ See e.g. Rachael Briggs, 'Normative Theories of Rational Choice: Expected Utility' in Edward N. Zalta (ed.), The Stanford Encyclopedia of Philosophy (Stanford University 2015).

${ }^{26}$ boyd (n 7).
} 
for example be the norm and therefore expected that Facebook experiments with our emotions and actions and allows hidden algorithmic audiences and so on. But it is crucial that Facebook also has a near practical monopoly on a range of social affordances that many in our contemporary society see as needs. Note that the issue is not just that new needs arise but that our judgment of our needs are malleable and relative to perceived opportunity costs. Some people for instance avoid seeking the healthcare they 'need' or fear registering to vote, as they-sometimes rightly - perceive their situational vulnerabilities and the dangers of the kinds of accessibilities and repercussions that might ensue from these actions.

As we shall see in the following, non-human animals employ various privacy-seeking measures to navigate precarious and deeply contextual and dynamic balances of needs and fears and thus optimize their survival possibilities. The question is to what extent we should think of human privacy needs along similar lines. Given such an analysis, practical measures of privacy can be seen as various ways of limiting the social perceptibility and 'actionability' of our behavior, and such measures therefore appear integral to our situated agency.

\section{Relational privacy and dynamic vulnerabilities}

To avoid pre-defining privacy in opposition to a public sphere, and rather attempt to first approach privacy in functional terms - as something we seek for some purpose - it is helpful to look at the dynamics of privacy seeking in other animals. A distinct advantage of looking to non-human animals is also that we avoid tying the analysis of our privacy needs to existing law and to property rights.

In a 1977 article 'The concept privacy and its biological basis', Klopfer and Rubenstein propose as a starting point that the degree and kind of privacy sought is a compromise between many forces both within and outside the organism. ${ }^{27}$ This is a simple but crucial point: whatever privacy is, it must be understood in the overall economy of needs and treats, vulnerabilities and powers. To some extent this can sound like Altman's psychological theory of privacy regulation as being an optimizing balance between concealing and disclosing, between social openness and closedness. ${ }^{28}$ However, the question is not simply about 'where to put the membrane,' but about a much more multidimensional dynamics. Most importantly for my purposes, the functionality of various privacy-ensuring measures is always intertwined with the existing affordance space, i.e. the possibilities and threats of the physical, social and cultural environment, as well as with the temporally shifting needs and references of the person/animal in question.

Note that I am not in the following analysis assuming that life in civil society is like non-human lives even in most respects. In a sense civil rights and regulatory policies are precisely ensuring that in many respects our worlds are not 'lawless' and our choices protected from the harshest prey-predator conditions. In civil society many new possibilities open up as most can trust that certain behaviors that 'in the wild' would expose vulnerabilities will not actually be exploited as that would be illegal or go against otherwise enforced social norms. However, this way of looking at human social practices precisely grants that the conditions influence needs, abilities and ensuing behavioral choices.

I suggest that we need to let go of the notion - propelled theoretically also by Hannah Arend $^{29}$ —of the 'civilized' public sphere as a place without predators, where all our vulnerabilities can be disregarded, where we can be 'free agents' without any conflicts of interest, which represents some kind of universal disembodied political point of view. Such theories obscure the situated biological and cultural conditions that precisely protect us

\footnotetext{
${ }^{27}$ Klopfer and Rubenstein (n 5).

${ }^{28}$ Irwin Altman, The environment and social behavior: privacy, personal space, territory, crowding (Brooks/Cole 1975).

${ }^{29}$ Hannah Arendt, The human condition (University of Chicago Press 1958/2013).
} 
against threats. We need privacy in various forms because we are agents among other agents. Because our socially perceived actions have social effects, and part of our action choice always will have to do with our estimation of our own perceptibility to others and the according social consequences. I call this our 'perceptible agency' ${ }^{30}$ and argue that this notion is pivotal for understanding both our various needs and tools of privacy. Note that our perceptible agency and its consequences are always relationally dependent on perceivers and contexts - and the proposal is that privacy should be understood relationally as well.

\subsection{Biology insight I: Relational privacy and needs beyond seclusion}

Interestingly, Klopfer and Rubenstein focus on the needs and contextual conditions when they describe the ranges of privacy dynamics one could expect in various animals:

Withdrawal, as physical privacy, may also represent the only way to achieve concealment; in turn, for many organisms, concealment may be the sole defense against predation. It is possible to develop models that allow prediction of cases in which a solitary, private mode of life would be favored over gregariousness. If a potential prey's privacy protection comes from hiding and if detection by a predator results in but a single kill, group formation may be the best strategy for the prey. Where the predator makes multiple kills, privacy is favored. Of course prey animals have other needs, too, that may conflict with the need for remaining watchful. Increased gregariousness increases the number of watchers, perhaps offsetting a decrease in concealment. ${ }^{31}$

What is made apparent by this description is firstly that there is a cost to isolation and withdrawal from the broader social and ecological world. Many needs cannot be dealt with in isolation. Secondly and very importantly, 'concealment' is not an absolute notion, but assumed to be relational. We are seeking concealment not in the abstract but from particular envisioned treats, in this case known kinds of predators. Thirdly, the functional goal of concealment as protection can sometimes be better obtained through group formation, as this kind of 'plain view anonymity' can afford many advantages in terms of both safety from predation as well as putting the animal in a social and physical affordance space where other needs can be fulfilled.

As representatives of a traditional public-private dichotomy, Warren and Brandeis suggested that the 'intensity and complexity of life' increased the 'need to be let alone' and for seclusion and 'retreat from the world'. ${ }^{32}$ We need to do things with limited social consequences beyond in relation to the intimate and trusted others that one seeks seclusion with. As we in 'civilized society' are met with increasingly complex demands and responsibilities, seclusion can indeed be needed. But what we are reminded of by Klopfer and Rubenstein is that whereas withdrawal can be useful, it cannot be our only tool particularly if we are social and cultural beings with complex needs and responsibilities. Staying in permanent seclusion is simply not an option for humans in the digital age. We must almost constantly enter our bodies and information into public or semi-public spaces of 'ambient surveillance'; hence the importance of the question of privacy in public. ${ }^{33}$

Therefore when we talk about privacy measures in public we need to think not simply about the particular forms of information, kinds of surveillances technologies, or whether

\footnotetext{
${ }^{30}$ Maria Brincker, 'Dynamics of perceptible agency - the case of social robots' [2016] Minds \& Machines 1.

${ }^{31}$ Klopfer and Rubenstein (n 5) 57.

${ }^{32}$ Warren and Brandeis (n 8).

${ }^{33}$ The ultimate seclusion for humans are our private thoughts. However as embodied beings our mental life depends on the publically accessible body. Warren and Brandeis and many legal theorists assume a problematic mind-body dualism that also permeates the privacy debate. See also supra, n 14 .
} 
there was 'consent'. Rather we need to think dynamically in terms of the needs, pressures and available affordances from the agent's point of view.

\subsection{Freedom, responsibility and the coercive power of surveillance}

As mentioned, humans have taken many measures to transform the lawless non-human predatorial conditions and create 'civilized spaces.' This makes many communal, pro-social and future-directed actions viable that would otherwise be reckless. We have done this through among other things the creation of social norms of responsibility and legal liability, making it possible to hold some accountable for certain actions. However, all such conditions are precarious and the predatorial and unsafe conditions have always been part of human cultures. Marginalized people carry the brunt of the risks, but everyone is vulnerable. As a matter of fact, I suggest that if we ignore this aspect of our social world, we shall fail to understand both our current loss and our distinct needs for relational privacy measures.

The issue of safety and responsibility is intimately linked to that of privacy in the current public debate, but too often in a problematic way. Often it is stressed like Plato does in his 'Myth of Gyges' that if we at will could be entirely invisible to others, we would all prove selfish and morally corrupt. ${ }^{34}$ This notion is generally the explicit rationale of current visible surveillance (e.g. CCTV's) that these deter people from breaking the law and thus support moral and responsible behavior. I have already praised the rule of law and I shall also not deny the efficiency of some kind of social watchful eye, but suggest — which might sound paradoxical to some - that actually our moral agency cannot survive too much surveillance and too micromanaging accountability measures. In other words, the question is: does a world of ambient surveillance actually undermine rather than ensure civilized and ethically responsible behavior?

The tension can already be seen in Bentham's own description of his notorious Panopticon structure ${ }^{35}$ - his architectural 'moral' exoskeleton. The idea was to create a utilitarian structure that would make people 'better' and instill the 'right behavior' by way of surveillance and discipline. The ingenious aspect of the architecture was in the asymmetry of visibility and power in the structure. Each subject would be in their individual cell (that is personally ID'ed and without anonymity) whereas the hierarchical disciplinary powers would be hidden within a non-transparent yet externally visible watchtower. Through this asymmetry of visibility one could instill a sense of being watched without the subjects actually being able to verify who occupied the tower at which times. Adding now the power asymmetry of action and the individually targeted disciplinary consequence, we have - as Foucault has highlighted - the perfect conditions for coercion: those surveyed and disciplined will by themselves 'choose' to carry out the will of the watchtower in the most efficient of ways. ${ }^{36}$ We know from Milgram's terrifying experiments, ${ }^{37}$ that even just introducing a watchful authority serves as a coercing scenario, and makes people carry out acts they consider wrong and feel bad doing. Interestingly, later studies have also shown that people report a lowered sense of agency and responsibility in such types of situations. ${ }^{38}$ Hence a paradoxical logic of Bentham's Panopticon is that the more minutely we are held responsible, the less responsible we feel. The crucial point is that if the structure imposes action decisions

\footnotetext{
${ }^{34}$ The myth is attributed to the voice of Plato's older brother Glaucon in The Republic. See Ferrari and Griffith, Plato: 'The Republic' (Cambridge University Press 2000).

35 Jeremy Bentham, Panopticon or the inspection house (Vol. 2) (1791).

${ }^{36}$ See Michel Foucault, Discipline and punish: The birth of the prison (Vintage 1977) for a fantastic analysis of the power asymmetries of Bentham's Panopticon as well as many other social institutions.

${ }^{37}$ Stanley Milgram, 'Behavioral study of obedience' (1963) 67 The Journal of abnormal and social psychology 371.

${ }^{38}$ Emilie A Caspar, Julia F Christensen, Axel Cleeremans and Patrick Haggard, 'Coercion changes the sense of agency in the human brain' (2016) 26 Current Biology 585.
} 
on us then they are precisely not ours. We might not choose the 'wrong' thing but this is obtained by us carrying out the choice made for us. Pervasive surveillance and disciplinary structures erode individuality, creativity, innovation and dissent and thus overall impede organic and democratic change. They erode the very feeling of responsibility and autonomy because they erode our actual autonomy, that is our processes of individual and communal self-legislation and self-formation.

Thus it seems that neither Gyges' ring nor Bentham's Panopticon are conducive to individual moral agency - as each ensures a corrupting asymmetry of power. In Bentham's Panopticon there is no privacy of action, no anonymity, and no ability to create privacy by strategically manipulating one's perceptibility agency, because the perceiver is hidden in the tower. With the ring of Gyges the diametrically opposite situation is created: an agent is made capable of acting in public without any exposure. It is in a sense a metaphoric disembodied agent that can create consequences without ever being perceptible in any aspect of their agency. Sadly it seems that we in our current technologically transformed public spaces are creating a lethal mix of these two asymmetric scenarios - of entirely hidden agents and overexposed agents. The question is whether true ethical agency needs a balance of partial exposure, and thus conditions of responsible yet autonomous and non-coerced action. Are we currently creating public spaces that undermine the independence of agency and reciprocal equality that morality requires? The ethical questions shall reemerge. But for now, we must keep in mind that asymmetries of perceptibility and traceable consequences - as we learn from other animals - are asymmetries of power. ${ }^{39}$

\subsection{Biology insight II: The relationality of perceptible agency}

This then brings us back to the point that the privacy-seeking behaviors of various animals depend on their knowledge of their predators and so on - and what I have called the relationality of their perceptible agency. How adaptive would camouflage, group formations and action timing be in the face of unknown predators with unexpected ways of sensing and knowing? For behavior to be adaptive it needs to show 'fitness', which profoundly depends on what aspects can be perceived and are thus socially efficacious. Of course most non-human animals do not need to understand their own perceptible agency, as their reliance might simply be on brute evolutionary trial and error feedback. However, to develop more flexible action repertoires and autonomous agency we need capacities to know and flexibly change our behavior according to changing kinds of social interactions. Through the notion of relational perceptible agency, we can see Goffman's ${ }^{40}$ lesson about the need to change social masks, also in the lives of many non-human animals. I suggest that it is an inescapable part of any social interaction that we are presented with a choice about how to present ourselves, how to let ourselves be perceived. It is through our perceptible agency that we control and shape social outcomes.

Note that my suggestion is not that we could or should have complete control over outcomes. That would be a return to the denial of our embodied, embedded and dependent natures. Compare here to the influence of gravity: it is an undeniable force in our lives, but as it is profoundly reliable and we have adapted to it, it supports our agency rather than prevents it. To perform an intentional throw, for example, we need to rely on our perception of the current target, and our habitual knowledge of the constrained workings of our body-in-theworld. ${ }^{41}$ The need to control our perceptible agency is much like limb movements. To

\footnotetext{
${ }^{39}$ Foucault (n 36).

${ }^{40}$ Erving Goffman, The presentation of self in everyday life (Harmondsworth 1978).

${ }^{41}$ For an interesting take on control and also what it means to be remote controlled and the role of perception and knowledge in it, see Daniel C Dennett, Elbowroom: the varieties of free will worth wanting (MIT Press 1984) Ch. 3 .
} 
perform an intentional social action we need to rely on knowledge of current social situations and the habitual workings of the social fabric. Modulating our perceptual agency-what we say and do, what we do not say or do-simply is our social action. Now just as we do not know all the precise or even possible consequences of throwing a ball (I think here both of motor variability and the notorious butterfly effect) we similarly do not know the exact effects and side effects of our social actions. Thus both the knowledge and control that I am talking about are modest. Much like it is hard to throw a ball if the target is jumping around, I suggest that we need a stable and relatively transparent social environment to judge what actions would be good, conducive to our goals as well as harmless to self and others and so on. Given that perceptible agency is truly relational, it is clear that even what aspects of our actions will have any social consequences at all is dependent on the kind of perceiver present. We for instance do not typically think that our pupil size or heart rate as perceptible, even as they are clearly there to be sensed in a shared space given the right kind of 'perceiver'.

To summarize, in terms of judging our situated options and vulnerabilities, the key parameters are about what kinds of 1) perceivers we are dealing with, 2) powers they have over us, and 3) responsibilities we have towards them. I shall argue that the current threat to privacy primarily consists in the vanishing of social spaces in which we have reliable knowledge and counter moves regarding these parameters. More and more spaces have, as boyd has highlighted, collapsed contexts and hidden audiences. ${ }^{42}$ Thus they become nontransparent and practically 'unbounded' from the perspective of action choice. We are in a sense left largely unable to rationally navigate our own worlds, and therefore in desperate need for re-structuring our relational perceptible agency. This relational and contextual situation, I suggest, is at the core of our 'practical' privacy needs.

\subsection{Biology Insight III: Anonymity and partial information as relational tools}

As mentioned, anonymity and partial information sharing have served as key tools in reassuring practical privacy protections in public. We have already discussed how opportunities for anonymity are rapidly vanishing, due to tools of identification and aggregation across contexts. We are moving freely in a new kind of Panopticon, where actions that before would happen in a sea of anonymity are now traceable to our person. Data-gathering tools along with unhampered algorithmic powers make such information highly categorizable and actionable; predictive and pre-emptive policing, surveillance-based personalized pricing schemes and so on are leaving us relationally incredibly vulnerable. These vulnerabilities often have to do with entirely unanticipated perceptibilities and consequences. As an example think of youngsters joking online - and entirely within the parameters of the law - realizing years down the line that they have been classified as 'high risk' and cannot get an affordable insurance or a mortgage. The Chinese 'citizen score' can be seen as merely a more explicit and aggregated version of much more varied global trends in this direction. Had the joking happened in a space allowing for anonymity, no such retroactive social consequences would have been possible. It would for most intents and purposes have been a bounded and knowable social affordance space.

Another important tool for relational privacy, which is related to but different from anonymity, is the ability to expose ourselves to a limited view as we act in public. Interestingly, Klopfer and Rubenstein talk about 'information management' in various nonhuman animals: ${ }^{43}$

\footnotetext{
${ }^{42}$ boyd (n 7).

${ }^{43}$ Compare to Westin's analysis of human cultural differences and how shared living spaces correlate with expressive and communicative privacy measures. Alan F Westin, Privacy and Freedom (Atheneum 1967), Ch. 1.
} 
Although extensive social bonding limits the amount of privacy that can be achieved by withdrawing from a group, other means of achieving privacy involving management of the flow of information can be used. Thus, while surrounded by neighbors, privacy can be achieved by preventing others from acquiring complete and accurate information about one's internal state or future intentions. ${ }^{44}$

Why is it so important to prevent others from complete and accurate information? It is to a large degree again about competitive advantage. They continue to describe how animal displays (perceptible agency) use more narrow and generalized signs to limit perceptibility of precise motivational states:

...the fact that the form of these signals is not associated with the organism's motivational state enables the sender to keep some information about itself or its intentions to itself. In essence, the organism has obtained some privacy. Thus although an animal may send an overt message, such as "I plan to escalate if you do not retreat," by means of a signal of typical form, it may also be hiding the fact that its present motivation level is low and that in reality it is on the verge of withdrawing. As a consequence, one of the functions of privacy is the withholding of information that otherwise might provide an opponent with a competitive advantage. ${ }^{45}$

This notion of privacy through partial communication is crucial and too often ignored in the privacy debate. ${ }^{46}$ It is a misunderstanding of our agentic dynamics to think of our behavior as simply either truthful or deceptive, as exposing or concealing. Each action as it is contextually chosen and produced is both revealing and concealing. If we lose this ability to conceal during exposure, I suggest we lose a key condition for autonomy.

There is a profound existential aspect to this vulnerability as it is about staying in realtime control of our actions. If too much is known, then another cannot just control me but directly act on the information and thus bypass my agency and my upcoming decisions. This is the threat that we are seeing now, as we are increasingly being judged and constrained by predictive algorithms, that is based on past data and according to indicators and categorizations determined by others. Our norms of linguistic conversation and civil interaction are largely based not only on actually limited access but also on social rules of what to leave out of the conversation. ${ }^{47}$ When we think of privacy needs as relational, we can also see how 'practical privacy' can be obtained by the - chosen or coerced - silence of others. ${ }^{48}$ However with aggregation and data mining, contexts are collapsed and these sorts of privacy measures - through controlled partial and relational exposure and situated trust and conventions - are largely being undermined. ${ }^{49}$

\footnotetext{
${ }^{44}$ Klopfer and Rubenstein (n 5).

${ }^{45}$ ibid

${ }^{46}$ Mark Zuckerberg for example argues — from behind the walls of his private compound — that if people share more, the world will become more open and connected. And a world that's more open and connected is a better world.' It is informative that the end goal here has to do with a faceless anonymous "world". This idea of "open and connected" as "better" smells like Bentham's rationale for the panopticon, and we might ask why he does not choose that openness for himself and his family, or-perhaps more importantly-for the actions and algorithms of his company. See Mark Zuckerberg, 'From Facebook, answering privacy concerns with new settings' (2011) http://www.washingtonpost.com/wp-

dyn/content/article/2010/05/23/AR2010052303828.html?tid=a inl.

${ }^{47}$ Interestingly, digital natives gave us the acronym “TMI” (Too Much Information), but see also Nagel (n 22) on how situational codes of conduct can let us retain certain functional privacy protections.

${ }^{48}$ For an analysis of the ways domestic privacy has been used to shelter abuse see Anita L Allen, Uneasy access: Privacy for women in a free society (Rowman \& Littlefield 1988).

${ }^{49}$ See boyd (n 3).
} 
The issue is that the moment of perspectival situated action is compromised. If I walk down the street, I might expect that neighbors can see me, but that would be a very limited exposure; only a fraction of the field of near and far affordances that I find myself in is available for my neighbors to perceive, even if I stop to small talk. Further, the implications this exposure would have for my future life, beyond shaping my neighbors' opinion, are limited. If on the other hand - perhaps via some over-sharing futuristic Google Glass - the neighbor was live-streaming and also had near-instant access to all sorts of information traceable to me, like web activity, real-time Fitbit data, dating profiles, past-predictive geolocations and so on, it would be a very different 'encounter'. Our judgment of what is relevant, what to do and say in that moment would be overpowered by what we might call the 'extra-situational' information and consequences. In other words, the 'integrity' of this context, as a local and situated social affordance space, would be severely compromised.

I have so far mentioned the issue of someone's agency being bypassed by too much information, but the same kind of data and algorithms are used to return partial and curated information back to me. Thus our perceived affordance space online is typically 'personalized.' Our options are not the same as those of others even as we go to the 'same' site. Thus we can be controlled from the outside not simply by having our choice bypassed but by someone controlling the world we perceive. ${ }^{50}$ Klopfer and Rubenstein write:

Acquiring accurate information about the environment is essential for survival, and the elaborate sensory capacities of most social animals attest to this fact. In general, an organism's relative fitness is related to its ability to correctly perceive and interpret its surroundings. ${ }^{51}$

We need accurate - or at least broadly reliable - information to actually optimize the choice we make. We should worry about so-called 'filter bubbles' and 'halls or mirrors' and the extremely coercive power such affordance space control has over our choice. ${ }^{52}$

To conclude our lessons from the animal world, let me re-iterate that information access and control is power. Privacy can in this context be seen as a tool of relational power and thus naturally be used by both predator and prey, and for good as well as for bad. Anita Allen writes:

Privacy aligns not with raw preference, but with prudent self-interest. The good of privacy is contingent. Sometimes we ought to go public when we prefer to hide; sometimes we ought to hide when we might prefer to go public. The important thing is that privacy, like information sharing, has a place in free society. Our moral interests include freedom from judgment, freedom to don masks, freedom to build and maintain reputations, and freedom to and from intimacy. ${ }^{53}$

We can now turn to our final analysis of autonomous and moral agency and its dependence on the possibility of relational privacy practices.

\section{Shared affordance spaces: Powers, frame shifts, morality and politics}

\footnotetext{
${ }^{50}$ Fake news has become a big concern in for instance the 2016 Brexit referendum and US elections. It is becoming easier to provoke, seed and control predictable responses by way of controlling what people perceive.

${ }^{51}$ Klopfer and Rubenstein (n 5).

${ }^{52}$ See e.g. Eli Pariser, The filter bubble: What the Internet is hiding from you (Penguin UK 2011); Julia Angwin, Dragnet nation: A quest for privacy, security, and freedom in a world of relentless surveillance (Macmillan 2014); Bruce Schneier, Data and Goliath: The hidden battles to collect your data and control your world (WW Norton \& Company 2015).

${ }^{53}$ Anita L Allen, Unpopular Privacy -What we must hide (OUP 2011) 195.
} 
Aristotle famously proclaimed: 'every art and every inquiry, and similarly every action and pursuit is thought to aim at some good. ${ }^{, 54}$ For our purposes the point is that 1) actions are organized in relation to some anticipated outcome, and 2) that from the perspective of the aiming agent this outcome is considered of value. We might be mistaken about the value, our powers to obtain it or the situation of choice such that knowing more we would regret the action. Aristotle proposes that an action is only truly voluntary if it is not due to 'external force' or 'owing to ignorance.' The question is if anticipated value, external force and ignorance need to be considered when judging whether surveillance changes and significantly hampers our abilities to act purposively in public. As discussed above, we take into account how our actions will be perceived, what others want and their relative power over us, and choose our actions with such social consequences in mind. Per implication our action choice also depends on our knowledge, trust and grasp of the motivations of the expected perceivers. ${ }^{55}$ It seems that surveillance not only makes the social side effects - and thus overall outcomes of actions - impossible to reliably anticipate, but further that the knowledge of surveillance can act as a severely coercing force in the moment of choice.

A further complication is that we often integrate far-reaching values - that are 'bigger than us,' beyond our current needs and with many stakeholders - into our action calibrations. We might want peace, social justice and a world structured such that we can take actions towards these goals. As perspectivally situated agents, we are able to fluidly shift our framework of action judgment and act with constantly changing outlooks depending on the needs and opportunities we perceive in ourselves and our near surroundings and the broader world. These constant relational calibrations of outlooks - or frame shifts - have often been ignored. Frame shifts have proved puzzling in disciplines like robotics and economics where agents' 'subjective preferences' are modeled without a view to their embodied, embedded and social dynamics and tensions. ${ }^{56}$ I suggest that most of our deliberative choices pertain to what perspective to take, that is to deciding the context of choice. Should I think longer-term or shorter-term, about what is better for me, family, friends, colleagues and/or everybody? And who is everybody? Does my action have consequences for its own conditions of possibility? This is actually almost always the case to some degree or other-everything is political, as the saying goes. Even in local 'self-regarding' or self-maintaining actions about what to eat and whether to exercise, future possibilities are always hovering in the background. ${ }^{57}$

It is a profound weakness of most theories of decision-making that they typically hold our capabilities to be stable and independent from the choice between preferences. ${ }^{58}$ Nothing could be further from the truth. Each action we perform transforms not only our world but also our and others' relation to it. They reshuffle or change the affordances available to us, both immediately and longer term. Through their notion of 'autopoiesis', Varela and Maturana have theorized the dynamics of contextual actions and self-maintenance in simple biological organisms ${ }^{59}$ - and profoundly transformed how many think about cognitive

\footnotetext{
${ }^{54}$ See Aristotle, The Nicomachean Ethics (David Ross tr, Library of Alexandria 1962) Book I, sec 1.

${ }^{55}$ For more on the development of the ability to include the perspectives of others see also Maria Brincker, 'Navigating beyond "here \& now" affordances — on sensorimotor maturation and "false belief" performance' (2014) 5 Frontiers in psychology (1433).

${ }^{56}$ For more on the classical frame problem in AI see e.g. Daniel C Dennett, 'Cognitive wheels: The frame problem of AI' in C Hookway (ed.), Minds, machines and evolution (CUP Archive 1986).

${ }^{57}$ Compare here to the neat distinctions of Arendt (n 29) between the self-maintaining actions of "labor" the world building actions of "work" and what she considers truly political "actions". For an attempt to blur these distinctions and bring our embodied natures back into the public space see James Mensch, 'Public Space and Embodiment' (2012) 12 Studia Phaenomenologica 211.

${ }^{58}$ See Briggs (n 25) and also Aristotle (n 54).

${ }^{59}$ Francisco G Varela, Humberto R Maturana and Ricardo Uribe, 'Autopoiesis: the organization of living systems, its characterization and a model' (1991) Facets of systems science (Springer US) 559.
} 
systems. ${ }^{60}$ Similar notions of how we continuously co-construct and shape our environments and ourselves as agents, need to be taken more seriously in the social sciences. ${ }^{61} \mathrm{We}$ are very passionate about changes to capabilities and environments - they can start and end wars.

Lasting changes to our situated possibilities constrain our entire future, and the evaluation of such lasting changes is a constant factor in our choices. My proposal is thus that we always have a profound interest and eye to the conditions of our own agency and the shifting power balances that come with shifting conditions for both ourselves and others.

One of the shortcomings of the traditional - and also Reidenberg's remodeledprivate-public dichotomy is that the very distinction is pushed outside the realm of action and in some sense made a priori and non-negotiable. This is too similar to traditional references to privacy-ensuring rules of 'decency' ${ }^{62}$ and 'civilized conduct' ${ }^{63}$ Even Nissenbaum's contextually bound norms of privacy only descriptively reflect how things are, rather than how we want them to be. If we instead think of norms as serving certain purposes, then they also naturally need to be negotiated between agents with diverging purposes. The negotiation of our public spaces and our shared affordance fields is political par excellence. My suggestion is that we need - to negotiate - ways of regulating such shared spaces such that they become reasonably bounded. The idea is that being able to understand and limit the (personally traceable) consequences of one's actions is pivotal to safe, responsible, autonomous and moral actions.

\section{Acting in unbounded contexts and regulating conditions of autonomy}

Now the question is how to act given the status quo. In our current world we are faced with the practical challenge of how we possibly could optimize our actions in surveyed environments, that is, environments that are in a sense per definition unbounded, and the relational consequences of which are unknowable. The key complication is that surveillance implies hidden agents that cannot be understood positively from within the perceptible environment. Thus in non-surveyed environments we accommodate our actions to our judgment of the nature, ability and motivations of somewhat exposed or known others - i.e. the perceived social affordances. But the question is how to interpret and act on the amorphous affordances of surveillance and data harvesting.

The core problem is that most surveyed environments precisely have clashing frames of consideration not allowing for an overall coherent calibration. The action choice cannot get off the ground unless we try to get some kind of coherent interpretation of the space going. I propose that generally we individually cope in one of the following ways:

- Denial: we pretend the surveillance is not there, and gamble on the disregard for the potential consequences of surveillance. I have nothing to hide/blind trust.

- Submission: we might, typically when disciplinary acts are perceived, focus on the assumed demand of an external authority, thus allowing a coercion of our choice away from its autonomous calibration given the local perceived social environment.

- Paranoia and paralysis: we might be overwhelmed by the uncertainty of the unbounded context. Seeing the watchtower but not knowing what counts as good or bad, laudable or punishable. The context is here deemed too dangerous for action, allowing only for retreat or paralysis.

\footnotetext{
${ }^{60}$ Embodied and enactive approaches to cognition have many precursors, but Varela and Maturana were critical in this revival of more dynamic and contextual ways of theorizing that is still unfolding. See e.g. Maria Brincker. Moving beyond mirroring-A social affordance model of sensorimotor integration during action perception (City University of New York 2010) Ch. 1.3.

${ }^{61}$ The interdisciplinary tradition of Critical Theory has already analyzed many such dynamics.

${ }^{62}$ Warren and Brandeis (n 8).

${ }^{63}$ Nagel (n 22).
} 
In our contemporary world, what many end up doing is the first and second. In many contexts we ignore the accessibility to unknown others and hope that it will be inconsequential - even when we to some extent know that this is unlikely. Interestingly, in this connection Klopfer and Rubenstein write that 'behavioral habituation, or the failure to respond overtly to repeated invasions of privacy, is in fact often seen among captive animals. ${ }^{64}$ Here of course we have cases of both lack of alternatives and lack of consequences of exposed vulnerabilities. In many employment situations the frantic inferno of 'indicators' and 'assessments' have imposed Panopticon-like environments of fearful and applause-seeking submissiveness. Overall, I worry that we currently are being habituated, and that the consequences of our exposed vulnerabilities and tame compliance will simultaneously be increasingly obvious and increasingly undermining of our individual and political autonomy - and thus hard to change.

\section{Summary: why we need contexts allowing for relational privacies}

Basically my suggestion is that we need to understand the nature of human agency and our need for relational privacy to better regulate privacy in public spaces. Below is an attempt to summarize some of the key components of action and how it relates to privacy.

A. Knowledge of relational affordances. Our capabilities to bring certain outcomes about are deeply embodied and contextually dependent. Voluntary action depends on various forms of knowledge. We need reasonably reliable expectations about our capabilities and of what the environment affords.

B. Value and calibrations of affordances. Agents act in relation not to singular affordances but to affordance spaces - choices are always situated calibrations of multiple interests and purposes given the perceived opportunities. To assess the value of potential outcomes we need to be able to expect certain consequences of our actions.

C. Perceptible agency. In social contexts the consequences of our actions are deeply dependent on which aspects of our behavior are perceived and by whom. This notion stresses the dynamic relationality of social action effects: it is not simply about what you do, but about how it is received. Appearances have real consequences.

D. Non-negotiable elements of contexts. Certain aspects of our bodies and environments are for practical purposes non-negotiable (such as gravity). We organize our actions around such expectations. In a sense our freedom and creativity relies on such constraints.

E. Coercion and power asymmetries. When other agents and culturally produced constraints appear as non-negotiable affordances due to power asymmetries, our actions are easily manipulated. Such control is typically the raison d'être of the perceived social constraint, and thus coerces enactment of the will of the architect.

F. Freedom and autonomy. Being a free agent means to be able to negotiate one's affordance space and choose and weigh the worthiness of actions from within the multiplicity of practical and moral concerns that make up one's situated perspective.

G. Morality and responsibility. The perspectival and situated nature of action does not make it inherently selfish or immoral. We can act according to our moral outlook and against personal gain or survival. But 'selfless' acts are still situated and chosen from a point of view, as some adopted moral outlook drives the choice.

H. Relational privacy. Intentional actions and choice calibrations rely on an ability to anticipate and create specific consequences. To avoid conditions of uncertainty and undue coercion, and to freely weigh and shift between complex needs and outlooks, we rely on relational privacy.

I. Unbounded contexts. Unregulated and thus unpredictable surveillance and data mining

\footnotetext{
${ }^{64}$ Klopfer and Rubenstein (n 5) 62.
} 
by unknown present and future perceivers and algorithms make for what I call unbounded contexts of action. Such contexts undermine some of the core conditions of our autonomous, deliberate and socially responsible agency.

J. Flexible privacy measures. As the function of privacy is relational, the means are flexible as long as they work to ensure some reliable limitation of actionable consequences beyond the current affordance space.

\section{Conclusion: relational privacy in public space}

This chapter suggests that to understand our needs and challenges of gaining privacy in public spaces, we must think of the conditions of our situated and embodied agency. However, the private-public dichotomy still profoundly shapes the privacy debate, and is perhaps preventing us from seeing the need for what I call relational privacy. Instead of focusing on access to bits of private information, I analyze how our actions are chosen in regard to a perceived affordance space. To choose appropriately we need to judge our contextual situation and to calibrate between complex needs, demands and outlooks. I argue that there is no such thing as disembodied, non-situated or non-perspectival action, and that this is crucial when we think about privacy.

Seclusion affords a situation of very limited social consequence, where we safely and easily can be vulnerable, spontaneous and tend to local intimate needs. However, public spaces traditionally also limit many social consequences as they allow limited perceptible agency. Thus the point is not just that semi-private places exists within some public spaces, but that all contexts for action are treated as limiting our relational exposure in some form or other. ${ }^{65}$ We seek — and can handle — relational publicity, we want exposure, perceptibility and recognition and social consequences of our actions, but not unbounded publicity. This is where our privacy needs and concerns come in.

We need privacy practices to be able to perform actions with limited and thus reasonably expected social consequences. This is particularly crucial when others are able to trace and thus act on these consequences in ways that relate directly to our future social standing. If it is correct that our need for privacy pertains to ways of limiting and controlling the social effects of our actions, then it is also expectable that privacy-ensuring measures can take many forms. Privacy practices should be versatile and adapt to the cultural, legal and technological context we are in. Note that with a relational approach a firm categorization of the private and public is unnecessary - it can be continuously negotiated by individuals and communities. What to reveal and to whom is precisely integral rather than external to our choices.

However we are currently creating technologically mediated public contexts in which it is very tricky to establish adaptive privacy limitations. Such contexts of surveillance and 'decision-making algorithms' are inherently unbounded in time and space, and even in terms of what can be perceived, and thus leave us with few options to keep our exposure limited and targeted. If we do not try to make such public contexts bounded, by for example regulating or taxing data collection, storage and secondary use, then we are likely to create new habits of excessive obfuscation or hiding in public, apathy or fear. Such habits, I suggest, would be mal-adaptive not only for our civic and democratic values but also for our basic autonomy and sense of personal responsibility.

\footnotetext{
${ }^{65}$ Thus we might even in Bentham's low-tech panopticon or with Orwell's Big Brother in practice take aspects of our agency to be imperceptible, until such assumptions are corrected by disciplinary feedback.
} 